\title{
Computed Tomography-Determined Sarcopenia Is a Useful Imaging Biomarker for Predicting Postoperative Outcomes in Elderly Colorectal Cancer Patients
}

\author{
Hailun Xie, $\mathrm{MS}^{1}$ \\ Yizhen Gong, $\mathrm{PhD}^{1}$ \\ Jiaan Kuang, MS \\ Ling Yan, MS \\ Guotian Ruan, MS' \\ Shuangyi Tang, $\mathrm{MD}^{2}$ \\ Feng Gao, MD' \\ Jialiang Gan, MD'
}

\begin{abstract}
Purpose
This study aimed to establish whether computed tomography (CT)-determined sarcopenia is a useful imaging biomarker for postoperative outcome in elderly colorectal cancer (CRC) patients, and construct sarcopenia-based nomograms to predict individual outcomes after surgery.
\end{abstract}

\begin{abstract}
Materials and Methods
CT imaging data of 298 elderly CRC patients who underwent surgery in 2012-2014 were retrospectively analyzed. Skeletal muscle mass was determined by CT, and sarcopenia was diagnosed based on the optimal cutoff value determined by X-tile program. The correlation between sarcopenia and risk of preoperative nutrition and postoperative complications was evaluated. A Cox proportional hazards model was used to determine survival predictors. Sarcopenia-based nomograms were developed based on multivariate analysis, and calibrated using concordance index and calibration curves.
\end{abstract}

\section{Results}

A total 132 patients (44.3\%) had sarcopenia based on the optimum cutoff values (29.9 $\mathrm{cm}^{2} / \mathrm{m}^{2}$ for women and $49.5 \mathrm{~cm}^{2} / \mathrm{m}^{2}$ for men). Sarcopenia was an independent risk factor for preoperative nutrition ( $\mathrm{p}<0.001$; odds ratio [OR], 3.405; 95\% confidence interval [Cl], 1.948 to 5.954 ) and postoperative complications ( $p=0.008$; OR, 2.192; $95 \% \mathrm{Cl}, 1.231$ to 3.903). Sarcopenia was an independent predictor for poor progression-free survival ( $p<$ 0.001 ; hazard ratio [HR], 2.175; 95\% $\mathrm{Cl}, 1.489$ to 3.179) and overall survival ( $p<0.001$; $\mathrm{HR}, 2.524 ; 95 \% \mathrm{Cl}, 1.721$ to 3.703). Based on multivariate analysis, we produced four nomograms that had better predictive performance.

\section{Conclusion}

CT-determined sarcopenia is a useful imaging biomarker for predicting preoperative nutritional risk, postoperative complications, and long-term outcomes in elderly CRC patients. The sarcopenia-based nomograms can provide a scientific basis for guiding therapeutic schedule and follow-up strategies.
Correspondence: Jialiang Gan, MD Department of Colorectal Anal Surgery, The First Affiliated Hospital of Guangxi Medical University, 6 Shuangyong Road, Nanning 530021, Guangxi, China Tel: $86-13878155172$

Fax: 86-5359801

E-mail: gj15172@163.com

Received November 15, 2019

Accepted April 16, 2020

Published Online April 17, 2020

\section{Key words}

Elderly colorectal cancer, CT-determined sarcopenia, Prognosis, Nutrition, Complication

\section{Introduction}

Colorectal cancer (CRC) is one of the malignancies with the highest morbidity and mortality worldwide. According to Global Cancer Epidemiological Statistics (GLOBOCAN) 2018, there are about 1.8 million new cases and 881,000 can- cer-related deaths worldwide, with the third highest incidence and second highest mortality rate worldwide, which accounts for about one tenth of all cancer cases and deaths [1]. In China, CRC has high morbidity and mortality rates and is one of the five most common tumors and one of the fourth most fatal tumors [2]. As the prevalence of CRC increases with age, the proportion of elderly CRC patients 
gradually increases with the acceleration of population aging [3]. According to the Surveillance, Epidemiology, and End Results database, approximately $70 \%$ of CRC patients are aged $>65$ years and about $40 \%$ are $>75$ years old. Although there has been a significant improvement in follow-up and treatment strategies for CRC, overall survival (OS) in elderly CRC patients remains low $[4,5]$. Surgery is still a powerful tool for treating CRC, but treatment of elderly patients can be challenging. These patients have a higher incidence of post-treatment complications and mortality [6]. In addition, they are also reported to be at risk of functional decline after treatment, with an incidence of $>60 \%$ in elderly patients [7]. Therefore, the prognostic assessment of elderly CRC patients is critical for guiding therapeutic schedule and follow-up strategies.

Recently, there has been increasing evidence that the gradual decline in nutrition and function is associated with disease progression and is one of the leading causes of poor treatment outcomes. Sarcopenia, as an objective nutrient assessment index independent of body mass index (BMI), is of increasing concern. Sarcopenia is a progressive and pervasive disease that is characterized by reduced skeletal muscle mass and function throughout the body, reduced physical ability, decreased quality of life, and increased risk of adverse events such as death [8]. Sarcopenia was first proposed by Rosenberg in 1989 [9]. It is worth noting that systemic inflammation is reported to be a risk factor for muscle loss, and muscle loss may be a useful external marker of systemic inflammatory response. Wallengren et al. [10] found that high NLR was associated with lower muscle mass. Malietzis et al. [11] found that patients with elevated C-reactive protein had increased muscle loss and that muscle loss was accelerated. Skeletal muscle mass reduction measured on computed tomography (CT) is considered the gold standard in diagnosing sarcopenia [12]. There is increasing evidence that preoperative CT-determined sarcopenia is associated with postoperative adverse short-term and long-term outcomes in CRC patients $[13,14]$. Most of the current research on sarcopenia in CRC is based on the general population. There are still no clear guidelines for treatment decisions in elderly CRC patients. Compared with general patients, elderly CRC patients are more susceptible to sarcopenia due to both cancer invasion and body aging. Thus, it is imprecise to infer the results from the general population to older patients who have sarcopenic changes due to aging and cancer.

Therefore, this study retrospectively analyzed the medical history of elderly CRC patients to explore the relationship of CT-determined sarcopenia with muscle loss and preoperative nutritional risk, postoperative complications and longterm prognosis in elderly CRC patients.

\section{Materials and Methods}

\section{Patients}

This retrospective study included data from the hospital records of 670 consecutive patients who underwent surgery for CRC at the First Affiliated Hospital of Guangxi Medical University, Nanning, China, between January 2012 and December 2014. We excluded eight patients were lost during follow-up, 342 patients aged $<60$ years when they underwent CRC resection, and 22 patients were not available for measurement of the area of skeletal muscle in the third lumbar spine by preoperative abdominal CT. In total, 298 elderly CRC patients were eligible for the study (Fig. 1). All patients included in this study did not receive preoperative chemoradiotherapy, and 118 patients received postoperative chemoradiotherapy.

\section{Survival follow-up}

Postoperative follow-up was performed every 3 months within 2 years after surgery and then every 3-6 months thereafter. The final follow-up visit occurred on September 1, 2019. The follow-up mainly included contacting the patient by telephone and investigating the patient in the clinic. The followup plan included physical examination, laboratory examination (tumor markers, blood routine, liver function test, kidney function test, etc.), imaging examination (flat, $\mathrm{CT}$ and magnetic resonance imaging) and endoscopy. Progressionfree survival (PFS) was determined as the duration between surgery and recurrence, death, or last follow-up. OS was determined as the duration between surgery and death or last follow-up.

\section{Data extraction}

The data collected included the following: general patient information, including sex, age, BMI (low, < 18.5; normal, $\geq 18.5$ and < 24; and high, 24), Nutrition Risk Screening 2002 (NRS-2002), American Society of Anesthesiologists (ASA) grade, Surgical method, and postoperative chemoradiotherapy; preoperative blood tests, including serum carcinoembryonic antigen (CEA) (normal, $<5 \mathrm{ng} / \mathrm{mL}$ ), lymphocyte counts and serum albumin concentration $(<35 \mathrm{~g} / \mathrm{L}$ was defined as hypoproteinemia); clinicopathological factors, including tumor location, pathological tumor stage (pT category), pathological node stage ( $\mathrm{pN}$ category), preoperative metastasis, pathological tumor-node-metastasis stage (pTNM stage), tumor perineural/vascular invasion, tumor differentiation, tumor pathological type, and tumor size; postoperative outcomes, including postoperative complications, Cla- 


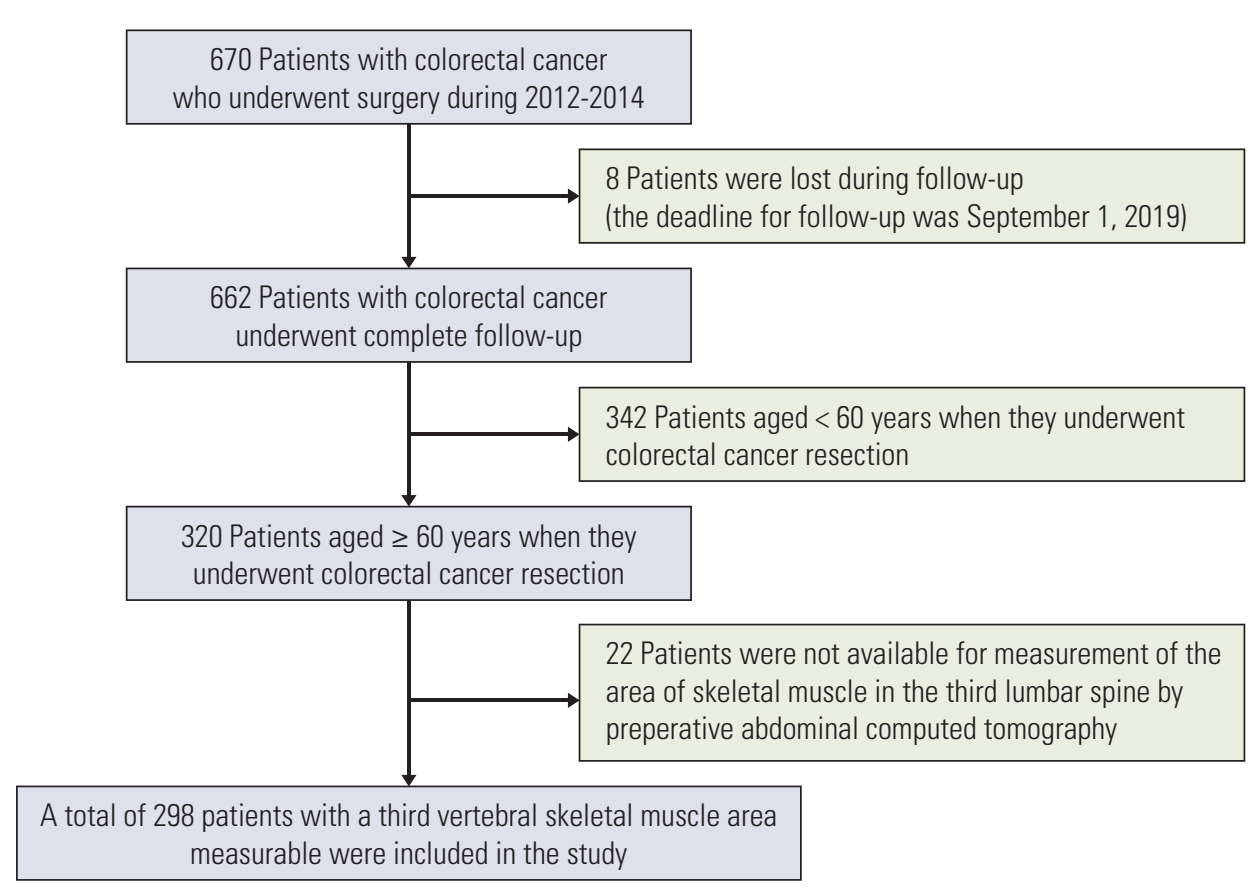

Fig. 1. The process of patients' inclusion and exclusion in elderly colorectal cancer patients.

vien-Dindo classification [15], length of hospital stays, PFS, and OS.

\section{Definition of skeletal muscle mass}

Abdominal CT was taken within 1 month before surgery. We selected a cross-sectional CT image of the third lumbar vertebra (L3) to estimate muscle mass and adjusted the CT threshold range from -29 to $150 \mathrm{HU}$ to separate skeletal muscle from other tissues. Psoas major, erector spine, quadratus psoas, transverse abdominis, internal and external oblique muscles, and rectus abdominis in the $\mathrm{L} 3$ region were measured. Then, referring to the BMI, the L3 muscle cross-sectional area calculated for each image was normalized by the square of the patient's height $\left(\mathrm{m}^{2}\right)$, and the skeletal muscle index $\left(\mathrm{SMI}, \mathrm{cm}^{2} / \mathrm{m}^{2}\right)$ was obtained [16].

\section{Statistical analysis}

The X-tile program [17] was used to determine the cutoff values of SMI for male and female patients, respectively. The chi-square test or $\mathrm{t}$ test was used to compare clinical variables. Kaplan-Meier analysis with the logarithmic rank test was used to construct a survival curve. Cox proportional hazards analysis was used to determine the prognostic factors of PFS and OS. Factors that were significant in multivariate analysis were used for nomograms, and the concordance index (C-index) and calibration curves were used to evaluate the accuracy of the nomograms. Statistical analysis was performed using IBM SPSS ver. 24.0 (IBM Corp., Armonk, NY) and R software ver. 3.5.3 (http:// www.r-project.org). A twotailed value of $p<0.05$ was considered to be statistically significant.

\section{Ethical statement}

This research was approved by the Ethics Committee of The First Affiliated Hospital of Guangxi Medical University of China and strictly complied with the provisions of the Helsinki Declaration (approval number: 2019 (KY-E-022)). All patients in this research have signed informed consent.

\section{Results}

\section{Clinicopathological factors}

Sex-specific cutoff values for SMI associated with OS were $29.9 \mathrm{~cm}^{2} / \mathrm{m}^{2}$ for women and $49.5 \mathrm{~cm}^{2} / \mathrm{m}^{2}$ for men. Using these cutoff values, $44.3 \%$ of patients were found to have sarcopenia. The clinicopathological features of the 298 elderly CRC patients are shown in Table 1. There were 197 (66.1\%) 


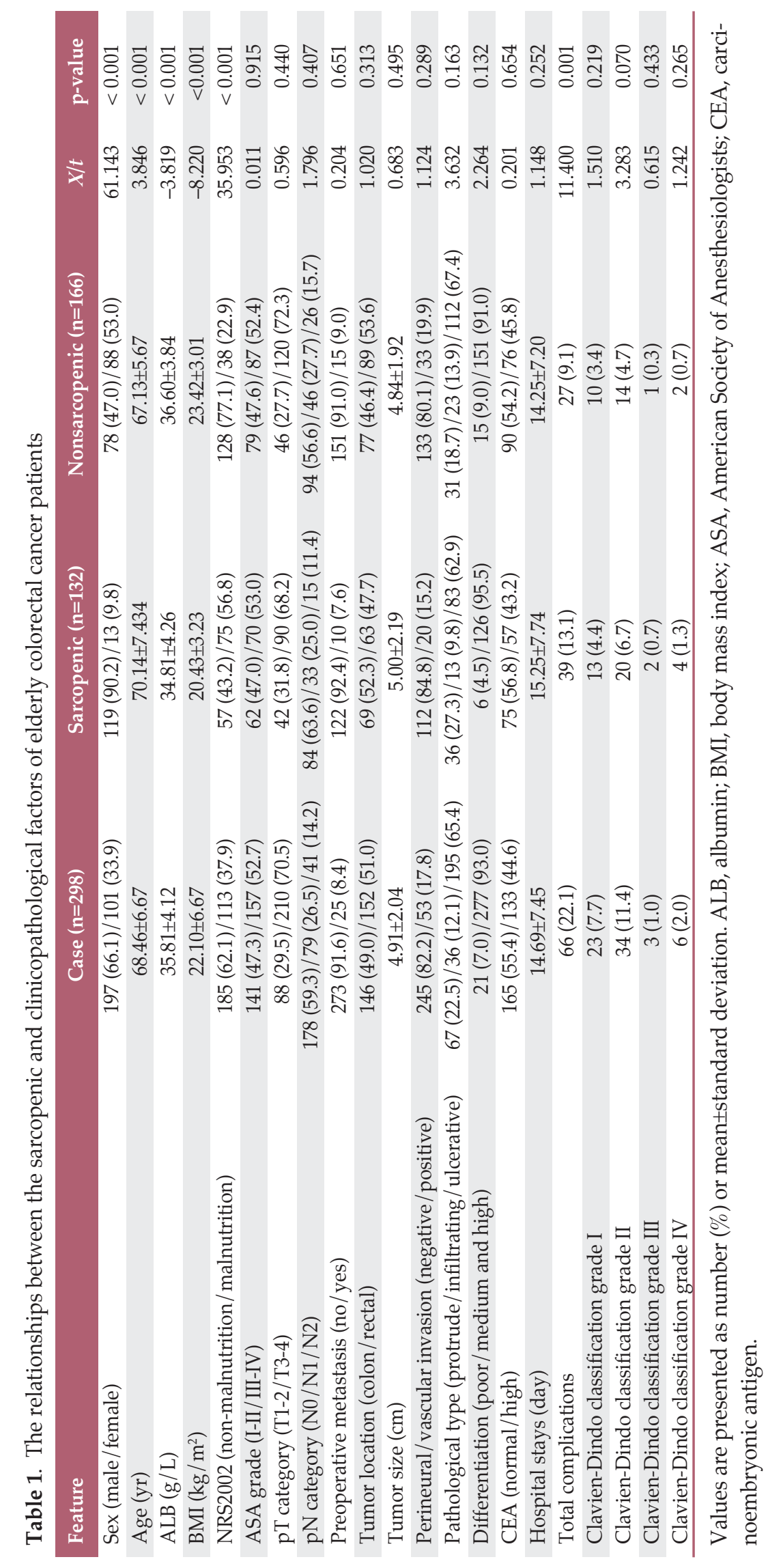


Table 2. Univariate and multivariate logistic regression analysis of factors associated with malnutrition in elderly CRC

\begin{tabular}{|c|c|c|c|c|}
\hline \multirow{2}{*}{ Feature } & \multicolumn{2}{|c|}{ Univariate analysis } & \multicolumn{2}{|c|}{ Multivariate analysis } \\
\hline & OR $(95 \% \mathrm{CI})$ & p-value & OR $(95 \% \mathrm{CI})$ & p-value \\
\hline Female sex & $0.920(0.560-1.512)$ & 0.743 & - & - \\
\hline Age ( $\geq 70 \mathrm{yr})$ & $1.537(0.951-2.483)$ & 0.079 & - & - \\
\hline $\operatorname{ALB}(\geq 35 \mathrm{~g} / \mathrm{L})$ & $0.319(0.195-0.520)$ & $<0.001$ & $0.418(0.244-0.716)$ & 0.001 \\
\hline BMI & & $<0.001$ & & 0.037 \\
\hline Low & 1.000 & & 1.000 & \\
\hline Normal & $0.233(0.115-0.472)$ & $<0.001$ & $0.381(0.179-0.813)$ & 0.013 \\
\hline High & $0.164(0.075-0.359)$ & $<0.001$ & $0.390(0.162-0.938)$ & 0.036 \\
\hline Sarcopenia (no) & $4.142(2.515-6.823)$ & $<0.001$ & 3.405 (1.948-5.954) & $<0.001$ \\
\hline ASA grade (I-II/ III-IV) & $0.688(0.430-1.101)$ & 0.119 & - & - \\
\hline pT category (T3-4) & $0.895(0.537-1.491)$ & 0.670 & - & - \\
\hline pN category & & 0.673 & & \\
\hline N0 & 1.000 & & - & - \\
\hline N1 & $0.783(0.450-1.361)$ & 0.385 & - & - \\
\hline N2 & $0.869(0.431-1.756)$ & 0.696 & - & - \\
\hline Preoperative metastasis (yes) & $2.237(0.978-5.116)$ & 0.056 & - & - \\
\hline Tumor location (colon/rectal) & $1.462(0.913-2.339)$ & 0.114 & - & - \\
\hline Tumor size $(<5 \mathrm{~cm} / \geq 5 \mathrm{~cm})$ & $1.694(1.031-2.784)$ & 0.038 & $1.505(0.856-2.645)$ & 0.155 \\
\hline Perineural/Vascular invasion (positive) & $0.733(0.390-1.378)$ & 0.335 & - & - \\
\hline Pathological type & & 0.590 & & \\
\hline Protrude & 1.000 & & - & \\
\hline Infiltrating & $0.741(0.322-1.706)$ & 0.481 & - & - \\
\hline Ulcerative & $0.750(0.427-1.319)$ & 0.318 & - & - \\
\hline Differentiation (medium and high) & $0.636(0.239-1.688)$ & 0.363 & - & - \\
\hline CEA (high) & $1.095(0.684-1.752)$ & 0.707 & - & - \\
\hline
\end{tabular}

CRC, colorectal cancer; OR, odds ratio; CI, confidence interval; ALB, albumin; BMI, body mass index; ASA, American Society of Anesthesiologists; CEA, carcinoembryonic antigen.

men and $101(33.9 \%)$ women. Their median age was 67 years (range, 60 to 92 years). Sixty-eight patients $(22.8 \%)$ were diagnosed with stage I CRC, $101(33.9 \%)$ with stage II, 103 $(34.6 \%)$ with stage III, and $26(8.7 \%)$ with stage IV. The median follow-up time was 62 months (range, 1 to 80 months). Seventy-four $(24.8 \%)$ and $124(41.6 \%)$ patients experienced recurrence and death, respectively.

\section{Association of sarcopenia with clinicopathological fac- tors}

The main features of elderly CRC patients with sarcopenia were male sex $(p=0.002)$, older age $(p<0.001)$, lower serum albumin ( $\mathrm{p}=0.002)$, lower BMI $(\mathrm{p}<0.001)$, and higher malnutrition risk $(\mathrm{p}<0.001)$. Other factors such as ASA grade, pT category, $\mathrm{pN}$ category, preoperative metastasis, tumor location, tumor size, perineural/vascular invasion, tumor differentiation, pathological type, and CEA were not related to the presence of sarcopenia (Table 1).
3. Risk factors for preoperative malnutrition and postoperative complications in elderly CRC patients

Currently, the most commonly used preoperative nutritional assessment method is NRS2002. All patients were assessed for NRS2002 before surgery, and 113 were diagnosed with malnutrition. In univariate logistic regression analysis, serum albumin ( $<<0.001)$, BMI $(\mathrm{p}<0.001)$, sarcopenia $(\mathrm{p}<0.001)$, and tumor size $(\mathrm{p}=0.038)$ were considered to be correlated with preoperative malnutrition. In multivariate logistic regression analysis, only serum albumin ( $\mathrm{p}=0.001$; odds ratio [OR], $0.418 ; 95 \%$ confidence interval [CI], 0.244 to 0.716), BMI ( $\mathrm{p}=0.037)$ and sarcopenia ( $<<0.001 ; \mathrm{OR}, 3.405$; 95\% CI, 1.948 to 5.954 ) were independent risk factors for predicting preoperative nutritional risk in elderly CRC patients (Table 2).

Sixty-six patients $(22.1 \%)$ suffered from postoperative complications, including anastomotic leak (4 cases), postoperative bowel obstruction (12 cases), wound problems ( 25 cases), pulmonary complications (12 cases), and other complications 
Table 3. Univariate and multivariate logistic regression analysis of factors associated with complications in elderly CRC

\begin{tabular}{|c|c|c|c|c|}
\hline \multirow{2}{*}{ Feature } & \multicolumn{2}{|c|}{ Univariate analysis } & \multicolumn{2}{|c|}{ Multivariate analysis } \\
\hline & OR $(95 \% \mathrm{CI})$ & p-value & OR $(95 \% \mathrm{CI})$ & p-value \\
\hline Female sex & $0.739(0.407-1.343)$ & 0.322 & - & - \\
\hline Age ( $\geq 70 \mathrm{yr})$ & $1.083(0.618-1.898)$ & 0.780 & - & - \\
\hline $\operatorname{ALB}(\geq 35 \mathrm{~g} / \mathrm{L})$ & $0.557(0.321-0.968)$ & 0.038 & - & - \\
\hline BMI & & 0.142 & & - \\
\hline Low & 1.000 & & - & \\
\hline Normal & $1.095(0.511-2.349)$ & 0.815 & - & - \\
\hline High & $0.552(0.225-1.353)$ & 0.194 & - & - \\
\hline Sarcopenia (yes) & $2.159(1.237-3.766)$ & 0.007 & $2.192(1.231-3.903)$ & 0.008 \\
\hline ASA grade (III-IV) & $1.395(0.802-2.429)$ & 0.239 & - & - \\
\hline pT category (T3-4) & $2.484(1.230-5.015)$ & 0.011 & $2.337(1.127-4.847)$ & 0.023 \\
\hline pN category & & 0.890 & & - \\
\hline N0 & 1.000 & & - & \\
\hline N1 & $1.167(0.622-2.187)$ & 0.631 & - & - \\
\hline N2 & $1.036(0.456-2.357)$ & 0.932 & - & - \\
\hline Preoperative metastasis (yes) & $2.583(1.102-6.058)$ & 0.029 & $1.988(0.811-4.874)$ & 0.133 \\
\hline Tumor location (colon/rectal) & $1.053(0.609-1.820)$ & 0.853 & - & - \\
\hline Tumor size $(<5 \mathrm{~cm} / \geq 5 \mathrm{~cm})$ & $0.906(0.517-1.588)$ & 0.731 & - & - \\
\hline Perineural/Vascular invasion (positive) & $1.332(0.673-2.638)$ & 0.410 & - & - \\
\hline Pathological type & & 0.139 & & - \\
\hline Protrude & 1.000 & & - & \\
\hline Infiltrating & $1.140(0.378-3.440)$ & 0.816 & - & - \\
\hline Ulcerative & $1.966(0.933-4.140)$ & 0.075 & - & - \\
\hline Differentiation (medium and high) & $0.541(0.209-1.402)$ & 0.206 & - & - \\
\hline CEA (high) & $1.129(0.652-1.954)$ & 0.665 & - & - \\
\hline Surgical method (open) & $2.035(1.141-3.629)$ & 0.016 & $1.693(0.929-3.084)$ & 0.086 \\
\hline
\end{tabular}

CRC, colorectal cancer; OR, odds ratio; CI, confidence interval; ALB, albumin; BMI, body mass index; ASA, American Society of Anesthesiologists; CEA, carcinoembryonic antigen.

(13 cases). There were 23 patients $(23.0 \%)$ with grade I complications, $34(9.6 \%)$ with grade II complications, three $(1.3 \%)$ with grade III complications, and six $(2.2 \%)$ with grade IV complications by Clavien-Dindo classification. Patients with sarcopenia had a higher incidence of total complications $(13.1 \%$ vs. $9.1 \%, \mathrm{p}=0.001)$ (Table 1$)$. Logistic regression analysis found that sarcopenia $(p=0.008$; OR, 2.192; 95\% CI, 1.231 to 3.903) was a useful indicator for predicting short-term postoperative complications in the elderly CRC patients (Table 3).

\section{Associations of sarcopenia with survival outcomes}

In univariate Cox proportional hazards analysis, patients with sarcopenia had significantly lower PFS and OS than those with non-sarcopenia, respectively $(\mathrm{p}=0.008$; hazard ratio [HR], 1.607; 95\% CI, 1.133 to 2.279 and $\mathrm{p}=0.001$; HR, $1.785 ; 95 \%$ CI, 1.252 to 2.545). After adjustment for clinicopathological factors, multivariate Cox proportional hazards analysis showed that sarcopenia was an independent risk factor for predicting long-term survival outcomes (PFS: $\mathrm{p}<$ 0.001 ; HR, 2.175; 95\% CI, 1.489 to 3.179 and OS: $\mathrm{p}<0.001$; HR, $2.524 ; 95 \%$ CI, 1.721 to 3.703 ) (Table 4).

In Kaplan-Meier analysis, patients in the sarcopenia group had poorer clinical outcomes in terms of PFS (48.5\% vs. $64.5 \%$, log-rank $p=0.007)$ and OS $(47.7 \%$ vs. $66.9 \%$, log-rank $\mathrm{p}=0.001$ ) than those in the non-sarcopenia group had (Fig. $2 \mathrm{~A}$ and $\mathrm{B}$ ). We also compared survival curves for each pathological TNM stage between the sarcopenia and non-sarcopenia groups. There were significant differences in both PFS (65.7\% vs. $87.9 \%, \log$-rank $\mathrm{p}=0.042)$ and OS $(62.9 \%$ vs. $90.9 \%$, log-rank $\mathrm{p}=0.010$ ) in TNM stage I (Fig. $3 \mathrm{~A}$ and D). Similar results were also found for PFS (57.8\% vs. $76.8 \%$, log-rank $\mathrm{p}=0.036)$ and OS (57.8\% vs. 80.4\%, log-rank $\mathrm{p}=0.010)$ in TNM stage III (Fig. 3B and E). However, there were no significant differences in TNM stage I (Fig. 3C and F) and IV patients (data not shown).

To correct confounding factors, we performed a subgroup 


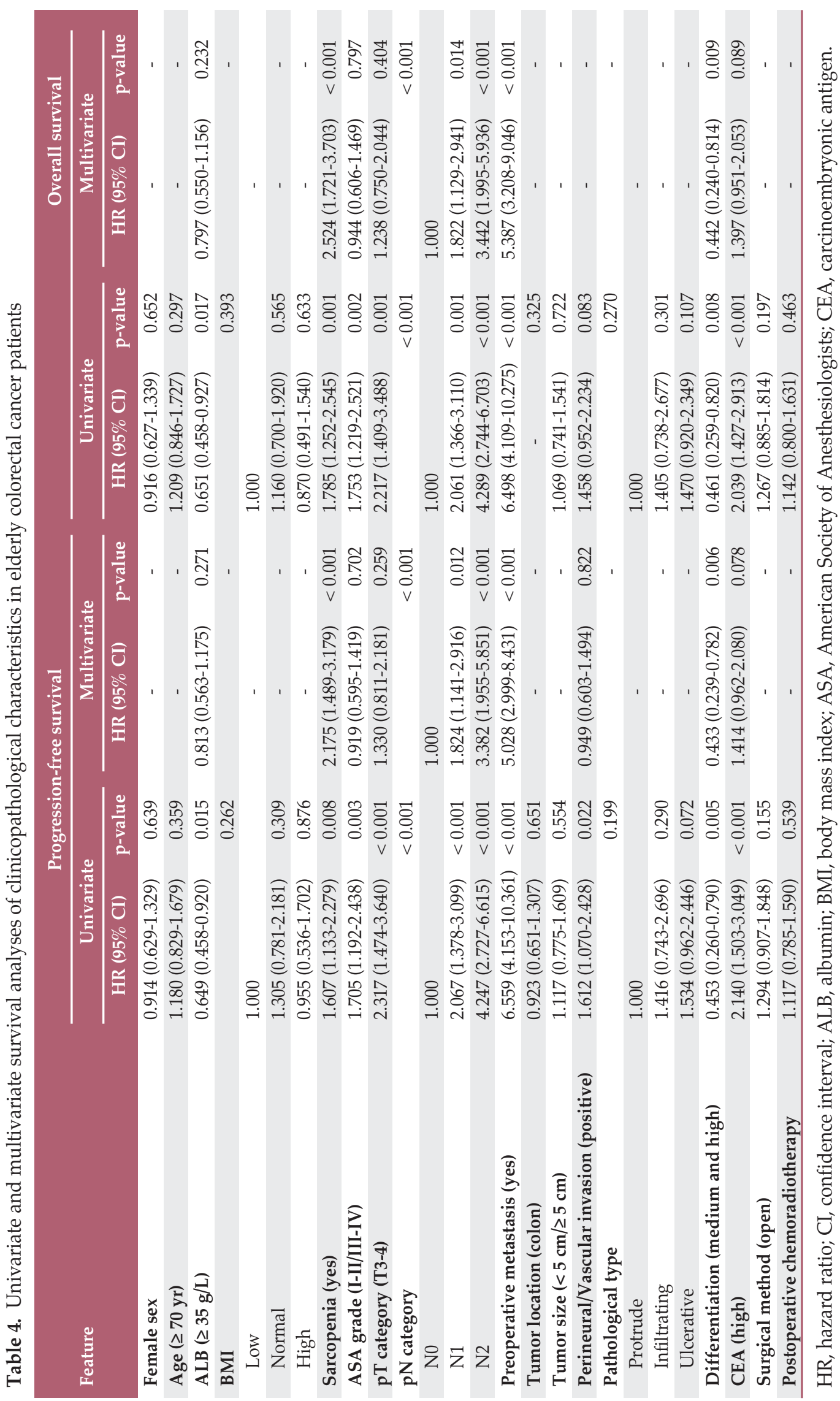




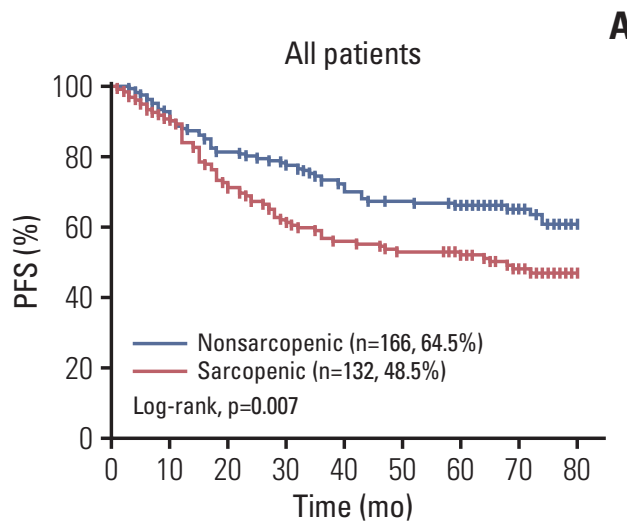

A

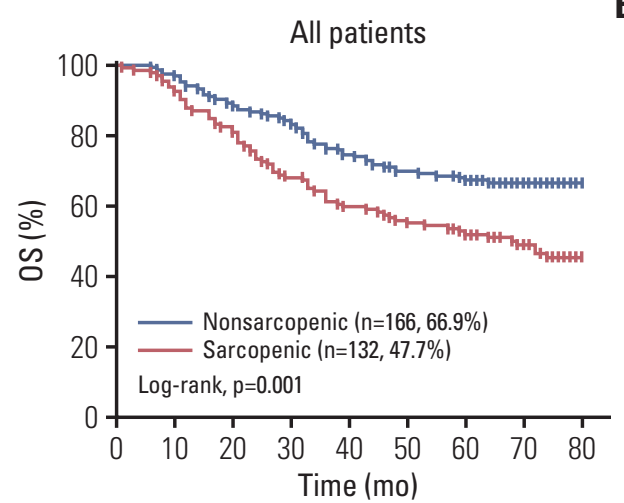

Fig. 2. Kaplan-Meier survival curves of sarcopenia and non-sarcopenia groups of all elderly colorectal cancer patients. (A) Kaplan-Meier progression-free survival (PFS) curves of all patients. (B) Kaplan-Meier overall survival (OS) curves of all patients.

multivariate analysis of each factor. 28 of the 35 subgroups indicated that sarcopenia was an independent risk factor for prognosis in PFS (Fig. 4A). Similarly, 28 of the 35 subgroups indicated that sarcopenia was an independent risk factor for prognosis in OS (Fig. 4B).

\section{Construction of sarcopenia-based nomograms}

Factors with $\mathrm{p}<0.05$ in univariate analysis were included in multivariate analysis. Based on multivariate analysis, we built four nomograms (A, nutritional risk model; B, complication risk model; C, PFS model; D, overall survival model) (Fig. 5). SMI was utilized as a continuous variable to improve the predictive accuracy of the nomograms, and the C-index value and calibration curves were used to evaluate the accuracy of the model. The $\mathrm{C}$-index for nutritional risk prediction was 0.738 (95\% CI, 0.679 to 0.797$)$, complication risk prediction was 0.635 ( $95 \%$ CI, 0.560 to 0.710 ), PFS prediction was 0.717 (95\% CI, 0.669 to 0.765 ), and OS prediction was 0.718 ( $95 \%$ CI, 0.672 to 0.764 ). The calibration curves for the probability of nutritional risk, complication risk, PFS, and OS demonstrated optimal consistency between the prediction by sarcopenia-based nomograms and actual observation (Fig. 6). By calculating the score of each factor, the preoperative nutritional risk, the risk of postoperative complications, and the PFS and OS of the elderly CRC patients could be predicted.

\section{Discussion}

The latest research suggests that sarcopenia in cancer patients is the result of local muscle inflammation. During tumor progression, tumor cells or surrounding cells are stimulated to produce tumor necrosis factor and interleukin-6 inflammatory cytokines [18]. Tumor necrosis factor inhibits skeletal muscle cell differentiation and promotes muscle atrophy [19]. Interleukin-6 can further reduce muscle protein synthesis by promoting protein degradation and reducing synthesis [20]. An increase in inflammatory cytokines can also lead to insulin resistance and muscle wasting by activating the ubiquitin proteasome proteolytic pathway, which further exacerbates systemic inflammation and muscle degeneration [21].

Gastrointestinal malignancies are often associated with the occurrence of dyscrasia. Studies have shown that about $80 \%$ of gastrointestinal cancer patients experience varying degrees of weight loss and muscle loss [22]. They are prone to obstruction, bleeding, and decreased food intake, which leads to increased glycogen decomposition and decreased synthesis of skeletal muscle, resulting in sarcopenia. In this study, approximately $44.3 \%$ of patients were considered to have sarcopenia. Patients with advanced age, hypoalbuminemia, low BMI, malnutrition were more likely to develop sarcopenia. We explored the relationship between sarcopenia and nutritional risk through logistic regression analysis and demonstrated that sarcopenia is a factor that can independently predict the preoperative nutritional risk of elderly CRC patients. As can be seen from the nutritional risk nomogram, sarcopenia has a higher impact on nutritional risk than serum albumin and BMI. Based on the above results, CTdetermined sarcopenia can be used as a new indicator of pre- 


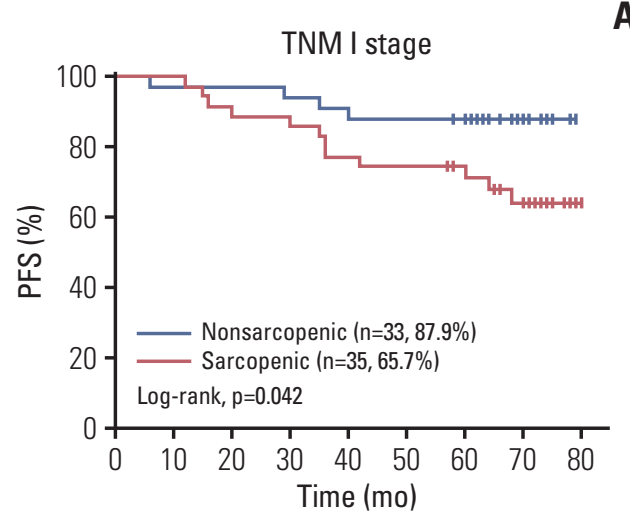

A
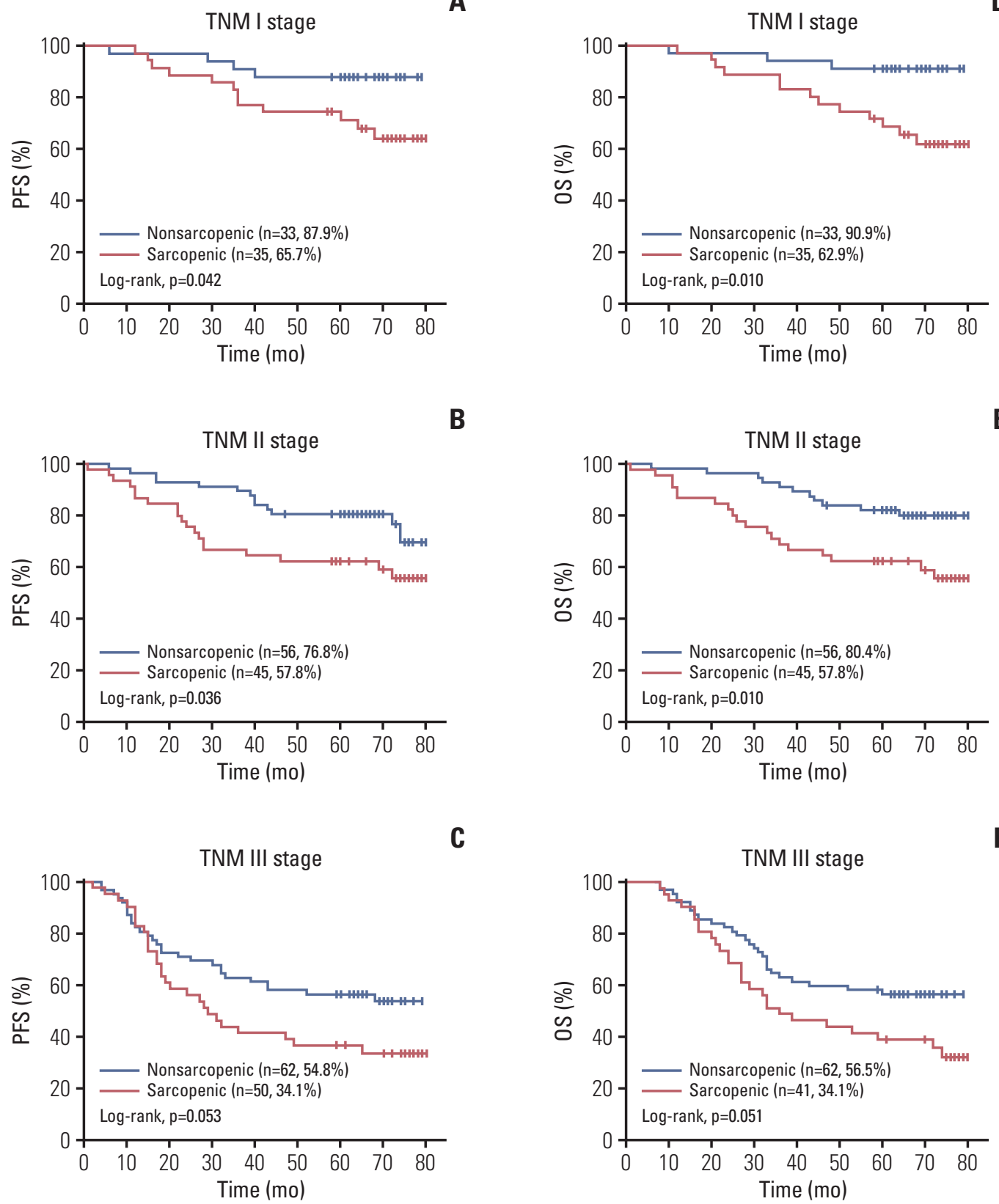

C

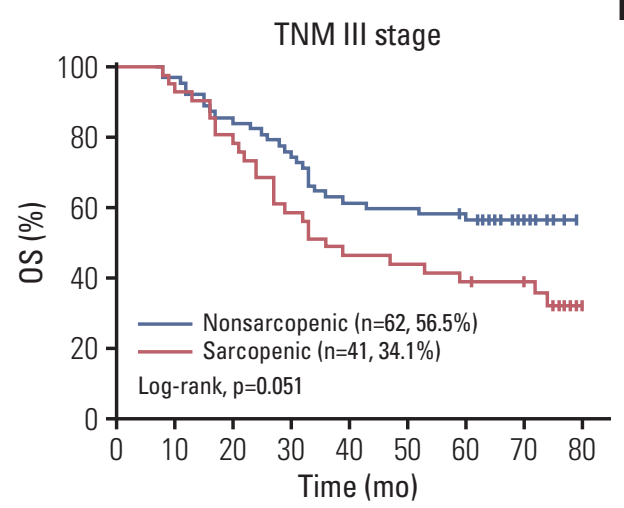

Fig. 3. Kaplan-Meier survival curves of sarcopenia and non-sarcopenia groups of elderly colorectal cancer patients based on each TNM stage. (A) Kaplan-Meier progression-free survival (PFS) curves of TNM I stage patients. (B) Kaplan-Meier PFS curves of TNM II stage patients. (C) Kaplan-Meier PFS curves of TNM III stage patients. (D) Kaplan-Meier overall survival (OS) curves of TNM I stage patients. (E) Kaplan-Meier OS curves of TNM II stage patients. (F) Kaplan-Meier OS curves of TNM III stage patients.

operative nutritional assessment. In elderly CRC patients with sarcopenia, approximately $16.8 \%$ had normal BMI and serum albumin. Although these patients appeared to be sufficiently healthy based on their appearance and preoperative examination and could undergo surgical resection, the true malnutrition status and unacceptable organ function were often ignored. In multivariate subgroup analysis, sarcopenia could be used to assess poor prognosis in patients with normal BMI and serum albumin. The above results indicated that CT-determined sarcopenia might be useful in identify- 
Sarcopenic (PFS)

\begin{tabular}{|c|c|c|c|c|}
\hline Subgroup & & Yes & No & HR $(95 \%$ CI) \\
\hline Sex & - $p \geq 0.05-p<0.05$ & & & \\
\hline Male & $i \vdash$ & 119 & 78 & 2.435 (1.459-4.064) \\
\hline Female & $\longmapsto$ & 13 & 88 & $2.543(1.052-6.146)$ \\
\hline Age (yr) & $i$ & & & \\
\hline$<70$ & $\longmapsto$ & 73 & 112 & $5.370(2.779-10.379)$ \\
\hline$\geq 70$ & $\mapsto$ & 59 & 54 & $2.375(1.272-4.435)$ \\
\hline ALB & 1 & & & \\
\hline Low & He-1 & 64 & 52 & $1.518(0.852-2.704)$ \\
\hline High & $\mapsto$ & 68 & 114 & $2.586(1.561-4.286)$ \\
\hline BMI & $\vdots$ & & & \\
\hline Low & $\mapsto$ & 39 & 7 & $1.514(0.276-8.306)$ \\
\hline Normal & $\mapsto$ & 74 & 90 & $2.520(1.508-4.213)$ \\
\hline High & $\longmapsto$ & 19 & 69 & 2.849 (1.033-7.859) \\
\hline ASA grade & i & & & \\
\hline$|-| \mid$ & $\mapsto 0-1$ & 62 & 79 & $1.477(0.800-2.726)$ \\
\hline III-IV & $1-1$ & 70 & 87 & $2.581(1.573-4.235)$ \\
\hline pT category & i & & & \\
\hline $\mathrm{T} 1-2$ & $\longmapsto$ & 42 & 46 & 4.386 (1.499-12.834) \\
\hline T3-4 & ${ }_{1}^{\prime} \mathrm{H}-1$ & 90 & 120 & $1.903(1.253-2.889)$ \\
\hline pN category & 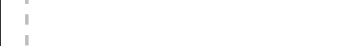 & & & \\
\hline NO & $\mapsto-\longrightarrow$ & 84 & 94 & $2.683(1.483-4.853)$ \\
\hline N1 & $\mapsto-1$ & 33 & 46 & $2.208(1.124-4.337)$ \\
\hline N2 & $\mathrm{H}$ & 15 & 26 & $1.525(0.674-3.450)$ \\
\hline Preoperative metastasis & i & & & \\
\hline No & $\mathrm{H}-\mathrm{H}$ & 122 & 151 & 2.101 (1.380-3.198) \\
\hline Yes & $\longmapsto$ & 10 & 15 & $2.507(0.611-10.288)$ \\
\hline Tumor location & 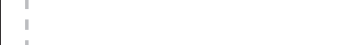 & & & \\
\hline Rectal & $1-1$ & 63 & 89 & $2.247(1.330-3.797)$ \\
\hline Colon & $1-1$ & 69 & 77 & $1.862(1.037-3.343)$ \\
\hline Tumor size $(\mathrm{cm})$ & $i$ & & & \\
\hline$<5$ & $\mapsto-1$ & 51 & 61 & $2.259(1.183-4.312)$ \\
\hline$\geq 5$ & $1 \mathrm{H}-1$ & 81 & 105 & $2.134(1.307-3.483)$ \\
\hline Perineural/Vascular invasion & ! & & & \\
\hline Negative & ${ }_{1}^{1} \mathrm{H}-1$ & 112 & 133 & $2.055(1.350-3.128)$ \\
\hline Positive & $\longmapsto$ & 20 & 33 & 2.401 (0.901-6.398) \\
\hline Pathological type & 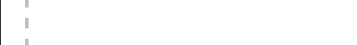 & & & \\
\hline Protrude & $\longrightarrow$ & 36 & 31 & $2.831(0.786-10.195)$ \\
\hline Lnfiltrating & $\longrightarrow \longrightarrow$ & 13 & 23 & $10.075(2.238-41.833)$ \\
\hline Ulcerative & ${ }_{1}^{\mathrm{t}} \mathrm{H}-\mathrm{C}$ & 83 & 112 & $1.915(1.222-2.999)$ \\
\hline Differentiation & 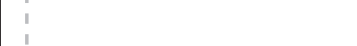 & & & \\
\hline Poor & $\longmapsto$ & 6 & 15 & $5.462(1.219-24.464)$ \\
\hline Medium and high & ite-1 & 126 & 151 & $2.347(1.282-2.860)$ \\
\hline CEA & i & & & \\
\hline Normal & : & 75 & 90 & $2.514(1.345-4.700)$ \\
\hline high & $H-1$ & 57 & 76 & $2.289(1.168-3.182)$ \\
\hline Surgical method & 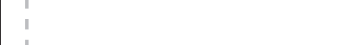 & & & \\
\hline Open & $1 \mathrm{H}-\mathrm{I}$ & 83 & 81 & $1.749(1.035-2.957)$ \\
\hline Laparoscopy & $\longmapsto$ & 49 & 85 & $3.374(1.833-6.209)$ \\
\hline Postoperative chemotherapy & $i$ & & & \\
\hline No & $1-1$ & 86 & 94 & $1.942(1.197-3.149)$ \\
\hline Yes & $\mapsto-1$ & 46 & 72 & $2.524(1.385-4.602)$ \\
\hline
\end{tabular}

Fig. 4. Subgroup multivariate survival analysis of sarcopenia in elderly colorectal cancer patients. (A) Subgroup multivariate progression-free survival (PFS) analysis of sarcopenia. (Continued to the next page) 
Sarcopenic (OS)

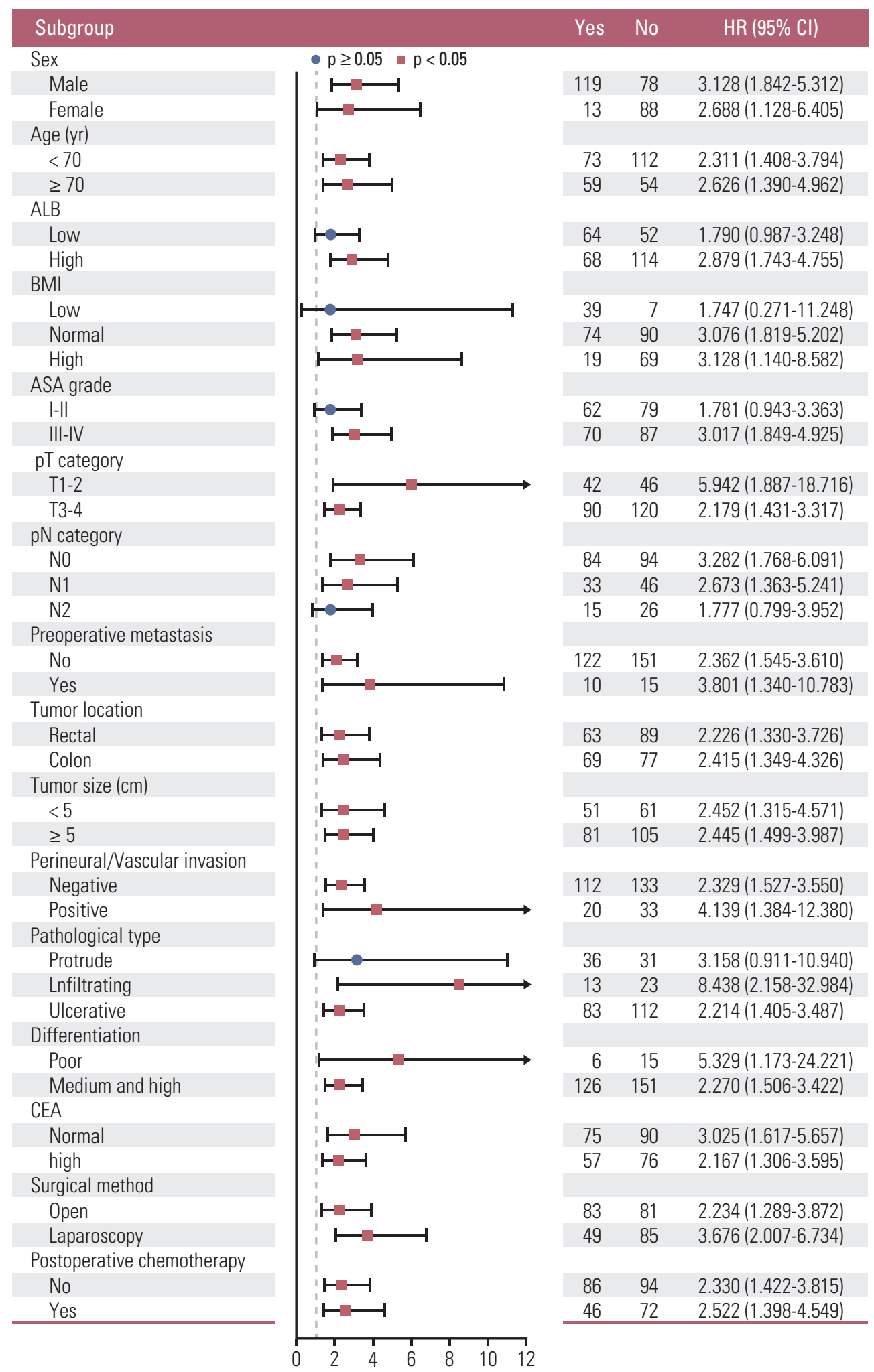

Fig. 4. (Continued from the previous page) (B) Subgroup multivariate overall survival (OS) analysis of sarcopenia. HR, hazard ratio; $\mathrm{CI}$, confidence interval; ALB, albumin; BMI, body mass index; ASA, American Society of Anesthesiologists; CEA, carcinoembryonic antigen. 
Points

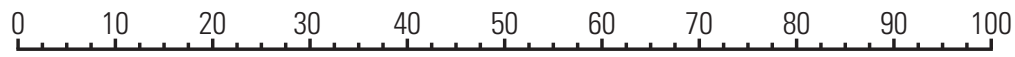

A

Skeletal muscle index

Serum albumin

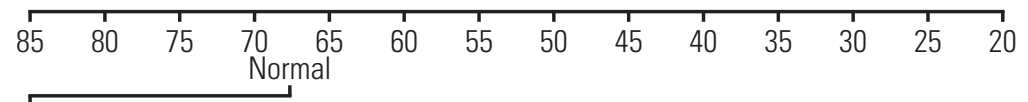

BMI

$$
\text { Low }
$$

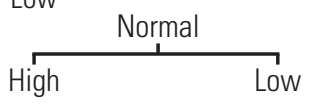

Total points

\begin{tabular}{lllllllll}
\hline & 20 & 40 & 60 & 80 & 100 & 120 & 140 & 160
\end{tabular}

Nutritional risk

$\begin{array}{llllllll}0.1 & 0.1 & 0.3 & 0.4 & 0.5 & 0.6 & 0.7 & 0.8\end{array}$

Points

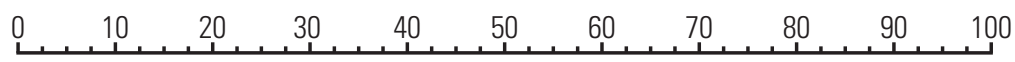

B

Skeletal muscle index

pT category

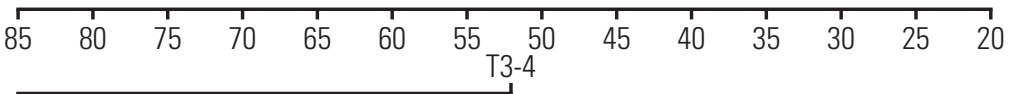

$\mathrm{T} 1-2$

Total points

\begin{tabular}{|rrrrrrrrrrrr}
\hline 0 & 20 & 40 & 60 & 80 & 100 & 120 & 140 & 160 \\
0.05 & & 0.1 & 0.15 & 0.2 & 0.25 & 0.3 & 0.35 & 0.4
\end{tabular}

Fig. 5. Construction of sarcopenia-based nomograms in elderly colorectal cancer patients. (A) Sarcopenia-based nomograms of nutritional risk. (B) Sarcopenia-based nomograms of complication risk. (Continued to the next page)

ing patients with occult malnutrition.

In the present study, about $22.1 \%$ of the elderly patients experienced varying degrees of postoperative complications. We determined that sarcopenia is an independent risk factor for postoperative complications, which is consistent with previous studies [14,23]. Sarcopenia leads to an increase in postoperative complications, which may be related to the following reasons. On the one hand, sarcopenia reflects malnutrition, and perioperative malnutrition in patients greatly increases the incidence of postoperative complications [24]. On the other hand, muscle mass and strength are key factors in maintaining body function, flexibility and vitality. Sarcopenia delays the early postoperative movement and early feeding time, which leads to impaired postoperative recovery [25].

Sarcopenia has been reported to have good prognostic value in many tumors, such as gastric cancer, hepatocellular carcinoma, and pancreatic cancer. However, different studies on the relationship between sarcopenia and long-term prognosis of CRC have yielded inconsistent results [23,26-28]. A multicenter study showed that sarcopenia is not associated with long-term prognosis, but is associated with short-term postoperative complications. The study showed that age had a greater impact than sarcopenia after the reduction of cancer load in vivo [27]. Since sarcopenia is related to human aging, skeletal muscle mass, skeletal muscle strength, and dysfunction undergo degenerative changes with age. In our study, we investigated the effects of sarcopenia on prognosis by screening elderly CRC patients aged $>60$ years, which to some extent reduced the bias caused by different ages. We showed that age was not an independent factor influencing survival outcomes, while sarcopenia was a good predictor of short- and long-term prognosis.

In survival analysis, we confirmed that sarcopenia is an independent risk factor for long-term outcomes, whether in PFS or OS. Besides, it can be seen from the survival nomograms that sarcopenia has a good impact efficiency, but comparing these risk-related factors, the effects of $\mathrm{pN}$ category and metastasis were greater than sarcopenia. It is well known that advanced tumor stages are associated with poor longterm prognosis of malignant tumors, but the prognosis of patients with the same stage is often different. We performed a stratified analysis of patients based on TNM staging and compared survival rates in patients with sarcopenia and non- 
Points

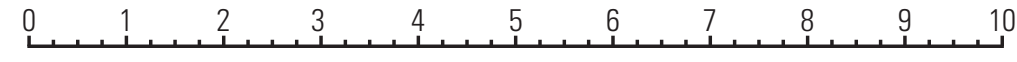

Skeletal muscle index

Differentiation

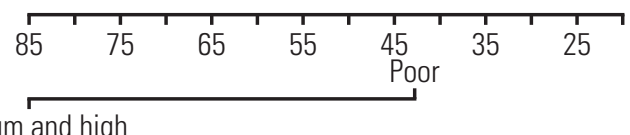

pN category

Preoperative metastasis

Total points

1-Year PFS

Medium and high
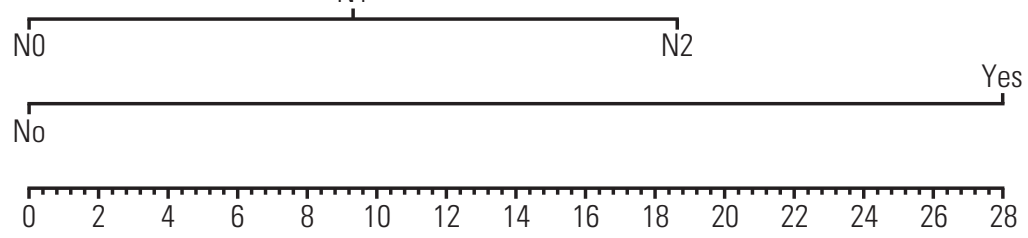

2-Year PFS

$\begin{array}{lllllllll}0.85 & 0.80 & 0.70 & 0.6 & 0.5 & 0.4 & 0.3 & 0.2 & 0.1\end{array}$

3-Year PFS

$\begin{array}{lllllllll}0.85 & 0.80 & 0.70 & 0.6 & 0.5 & 0.4 & 0.3 & 0.2 & 0.1\end{array}$

4-Year PFS

$\begin{array}{lllllllll}0.85 & 0.80 & 0.70 & 0.6 & 0.5 & 0.4 & 0.3 & 0.2 & 0.1\end{array}$

5-Year PFS

$\begin{array}{lllllllll}0.85 & 0.80 & 0.70 & 0.6 & 0.5 & 0.4 & 0.3 & 0.2 & 0.1\end{array}$

$\begin{array}{lllllllll}0.85 & 0.80 & 0.70 & 0.6 & 0.5 & 0.4 & 0.3 & 0.2 & 0.1\end{array}$

Points

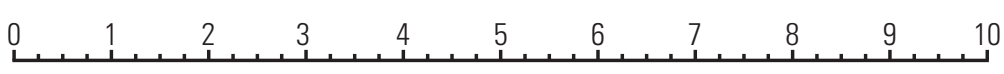

D

Skeletal muscle index

Differentiation

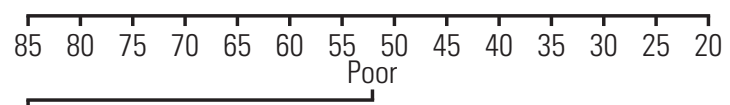

pN category

Medium and high

Preoperative metastasis
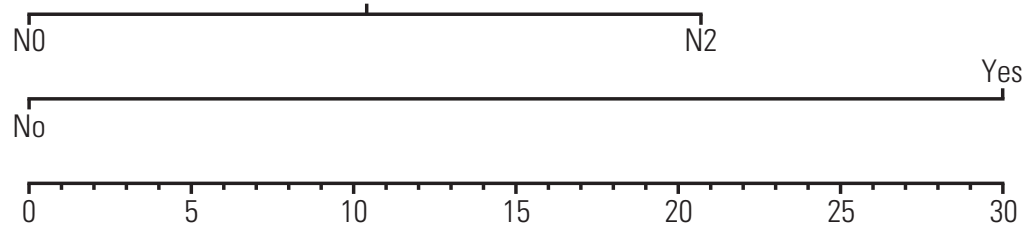

1-Year OS

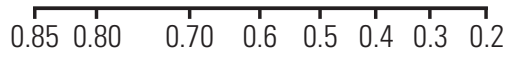

2-Year OS

$$
\begin{array}{lllllllll}
0.85 & 0.80 & 0.70 & 0.6 & 0.5 & 0.4 & 0.3 & 0.2 & 0.1
\end{array}
$$

3-Year OS

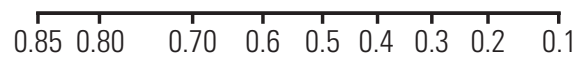

4-Year OS

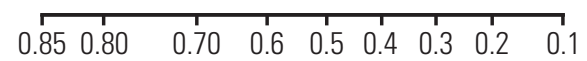

5 -Year OS

$$
\begin{array}{lllllllll}
0.85 & 0.80 & 0.70 & 0.6 & 0.5 & 0.4 & 0.3 & 0.2 & 0.1
\end{array}
$$

Fig. 5. (Continued from the previous page) (C) Sarcopenia-based nomograms of progression-free survival (PFS) (D) Sarcopenia-based nomograms of overall survival (OS). BMI, body mass index. 
A
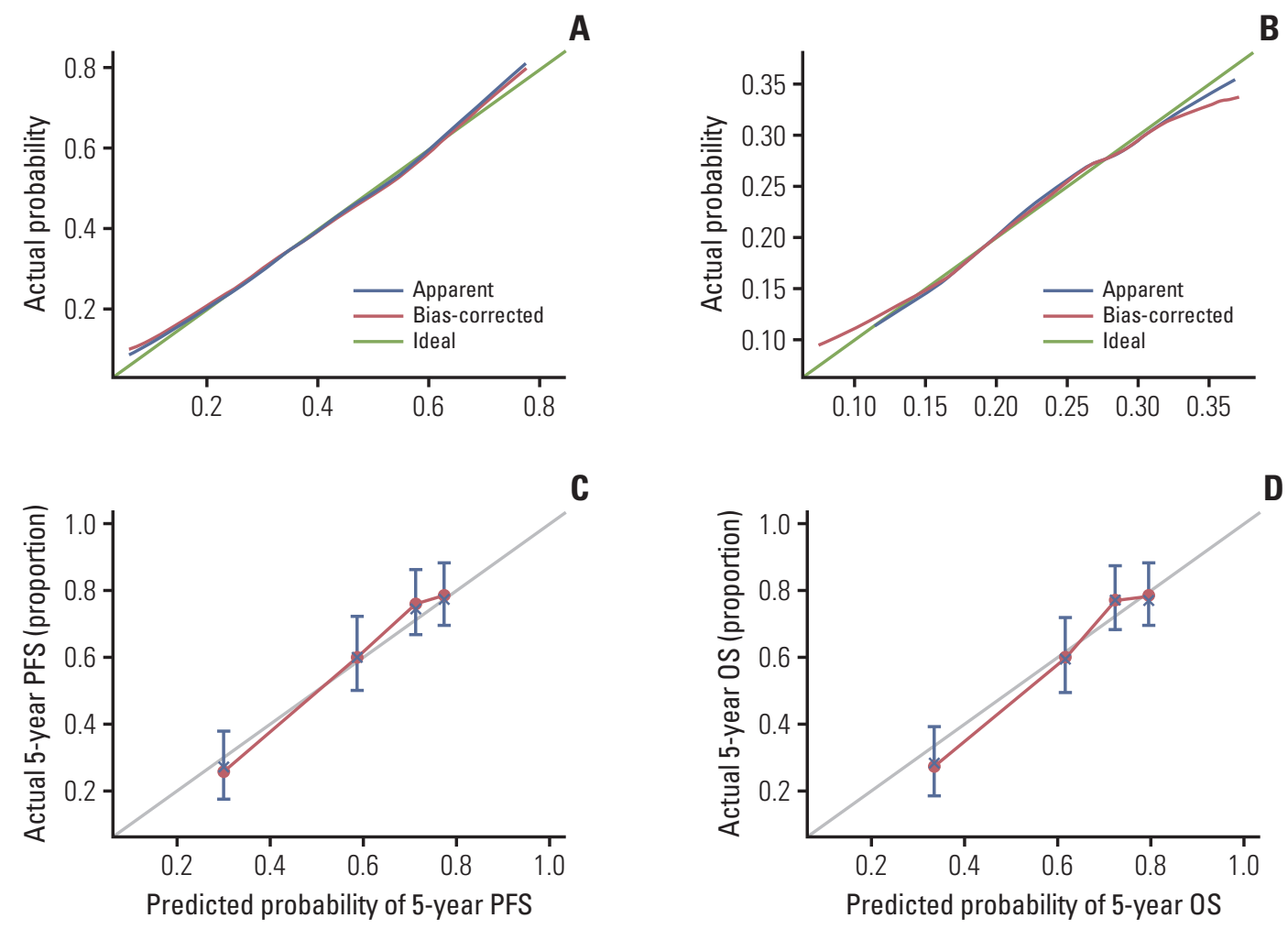

Fig. 6. The calibration curves for predicting nutritional risk (A), complication risk (B), progression-free survival (PFS) (C), and overall survival (OS) (D) in elderly colorectal cancer patients. The $\mathrm{X}$ axis presents the predicted probability and the $\mathrm{Y}$ axis shows the actual probability. The calibration lines fit along with the 45 reference.

sarcopenia. The results showed that OS and PFS of the sarcopenia group were significantly lower than those of the non-sarcopenia group in TNM stage I and II, but not in stage IIII and IV. Although in TNM III stage, Patients with sarcopenia still tend to have a poor prognosis, but it is not the main factor affecting prognosis. This may be because, in the early stage of the tumor, sarcopenia has a large impact on the prognosis of the patient. As the tumor progresses, the tumor invasion and metastasis gradually occupy the main position.

Nomograms are considered to be a direct tool for individualized risk assessment for each patient. We constructed four novel and effective nomograms based on sarcopenia for individualized assessment of preoperative nutritional risk, postoperative complications, PFS, and OS in elderly CRC patients. It is worth noting that the results of the C-index and the calibration chart confirm that the nomograms have good prediction accuracy. These four sarcopenia-based nomograms, combining clinically easy-to-use clinicopathological factors, are viable and reliable risk prediction tools for individualized prediction of elderly CRC patients, which may contribute to individualized postoperative follow-up and treatment options. Using sarcopenia-based nomograms to help screen high-risk patients can facilitate early treatment interventions, which is beneficial to improve the prognosis of elderly CRC patients. Resistance training [29], and antiinflammatory drugs [30] have been reported to be safe and effective in maintaining and increasing muscle mass and function in cancer patients. Early application of these measures can improve the quality of life and achieve longer survival.

CT is considered clinically as an accurate method for assessing skeletal muscle mass. It provides important quantitative information about muscle composition and distribution through high-quality images, spatial accuracy, and location features, as well as the ability to measure muscle mass from a cross-section of an abdomen. Before CRC surgery, an abdominal CT scan or enhanced examination is usually performed to evaluate tumor staging. If the L3 skeletal muscle mass is measured at the same time, it will not increase any cost, which can reduce the burden on the patient. Other detection methods for skeletal muscle reduction include dual-energy $\mathrm{X}$-ray absorption, nuclear magnetic resonance imaging, and bioelectrical impedance. Due to the complexity of the other methods, the testing process requires additional 
patient inspection costs. The prospects for clinical application are far from good than CT examinations. However, the measurement of skeletal muscle mass is still not a routine measurement item in CT examination. More studies are still needed to elucidate the importance of preoperative skeletal muscle mass measurement.

There were some limitations to our study. First, as a single-center and retrospective study, there were problems such as insufficient sample size and selection bias. Second, the definition of sarcopenia was based on muscle mass on $\mathrm{CT}$, and we did not investigate muscle strength or function. Based on the overall survival and overall survival time, this study used the X-tile program to determine the optimal cutoff value, which may have certain subjectivity. However, there are no clear diagnostic criteria for CT-determined sarcopenia in elderly CRC patients. In our opinion, this method can provide some reference value for subsequent research. Finally, sarcopenia-based nomograms are designed based on a limited population. Although the calibration curve suggested that they have good effectiveness, it is not verified by other independent teams. Therefore, the validity and practicability of the nomograms should be verified in the multicenter and large-sample population in the future.

This study is believed to be the first to explore the relationship between sarcopenia and preoperative nutrition, postoperative complications, and long-term outcomes of elderly CRC patients. The results suggest that CT-determined sarcopenia is a useful imaging biomarker for predicting preoperative nutritional risk, postoperative complications, and long-term outcomes in elderly CRC patients. The sarcopenia-based nomograms can provide a scientific basis for guiding therapeutic schedule and follow-up strategies.

\section{Conflicts of Interest}

Conflicts of interest relevant to this article was not reported.

\section{Acknowledgments}

This research was supported by the 2019 Innovation Project of Guangxi Graduate Education (JGY2019052).

\section{References}

1. Bray F, Ferlay J, Soerjomataram I, Siegel RL, Torre LA, Jemal A. Global cancer statistics 2018: GLOBOCAN estimates of incidence and mortality worldwide for 36 cancers in 185 countries. CA Cancer J Clin. 2018;68:394-424.

2. Chen W, Zheng R, Baade PD, Zhang S, Zeng H, Bray F, et al. Cancer statistics in China, 2015. CA Cancer J Clin. 2016;66:11532.

3. Arnold M, Sierra MS, Laversanne M, Soerjomataram I, Jemal A, Bray F. Global patterns and trends in colorectal cancer incidence and mortality. Gut. 2017;66:683-91.

4. van Steenbergen LN, Elferink MA, Krijnen P, Lemmens VE, Siesling S, Rutten HJ, et al. Improved survival of colon cancer due to improved treatment and detection: a nationwide population-based study in The Netherlands 1989-2006. Ann Oncol. 2010;21:2206-12.

5. Quaglia A, Tavilla A, Shack L, Brenner H, Janssen-Heijnen M, Allemani $\mathrm{C}$, et al. The cancer survival gap between elderly and middle-aged patients in Europe is widening. Eur J Cancer. 2009;45:1006-16.

6. Verweij NM, Schiphorst AH, Maas HA, Zimmerman DD, van den Bos F, Pronk A, et al. Colorectal Cancer Resections in the Oldest Old Between 2011 and 2012 in The Netherlands. Ann Surg Oncol. 2016;23:1875-82.

7. Hamaker ME, Prins MC, Schiphorst AH, van Tuyl SA, Pronk A, van den Bos F. Long-term changes in physical capacity after colorectal cancer treatment. J Geriatr Oncol. 2015;6:153-64.

8. Cruz-Jentoft AJ, Baeyens JP, Bauer JM, Boirie Y, Cederholm T,
Landi F, et al. Sarcopenia: European consensus on definition and diagnosis: Report of the European Working Group on Sarcopenia in Older People. Age Ageing. 2010;39:412-23.

9. Rosenberg IH. Sarcopenia: origins and clinical relevance. J Nutr. 1997;127(5 Suppl):990S-1S.

10. Wallengren O, Iresjo BM, Lundholm K, Bosaeus I. Loss of muscle mass in the end of life in patients with advanced cancer. Support Care Cancer. 2015;23:79-86.

11. Malietzis G, Currie AC, Johns N, Fearon KC, Darzi A, Kennedy RH, et al. Skeletal muscle changes after elective colorectal cancer resection: a longitudinal study. Ann Surg Oncol. 2016; 23:2539-47.

12. Mourtzakis M, Prado CM, Lieffers JR, Reiman T, McCargar LJ, Baracos VE. A practical and precise approach to quantification of body composition in cancer patients using computed tomography images acquired during routine care. Appl Physiol Nutr Metab. 2008;33:997-1006.

13. Shachar SS, Williams GR, Muss HB, Nishijima TF. Prognostic value of sarcopenia in adults with solid tumours: A metaanalysis and systematic review. Eur J Cancer. 2016;57:58-67.

14. Simonsen C, de Heer P, Bjerre ED, Suetta C, Hojman P, Pedersen BK, et al. Sarcopenia and postoperative complication risk in gastrointestinal surgical oncology: a meta-analysis. Ann Surg. 2018;268:58-69.

15. Dindo D, Demartines N, Clavien PA. Classification of surgical complications: a new proposal with evaluation in a cohort of 6336 patients and results of a survey. Ann Surg. 2004;240:205- 
13.

16. Lieffers JR, Bathe OF, Fassbender K, Winget M, Baracos VE. Sarcopenia is associated with postoperative infection and delayed recovery from colorectal cancer resection surgery. $\mathrm{Br}$ J Cancer. 2012;107:931-6.

17. Camp RL, Dolled-Filhart M, Rimm DL. X-tile: a new bioinformatics tool for biomarker assessment and outcome-based cut-point optimization. Clin Cancer Res. 2004;10:7252-9.

18. Baracos VE. Regulation of skeletal-muscle-protein turnover in cancer-associated cachexia. Nutrition. 2000;16:1015-8.

19. Fearon KC, Glass DJ, Guttridge DC. Cancer cachexia: mediators, signaling, and metabolic pathways. Cell Metab. 2012;16: 153-66.

20. Rong YD, Bian AL, Hu HY, Ma Y, Zhou XZ. Study on relationship between elderly sarcopenia and inflammatory cytokine IL-6, anti-inflammatory cytokine IL-10. BMC Geriatr. 2018;18: 308 .

21. Srikanthan P, Hevener AL, Karlamangla AS. Sarcopenia exacerbates obesity-associated insulin resistance and dysglycemia: findings from the National Health and Nutrition Examination Survey III. PLoS One. 2010;5:e10805.

22. Tsoli M, Robertson G. Cancer cachexia: malignant inflammation, tumorkines, and metabolic mayhem. Trends Endocrinol Metab. 2013;24:174-83.

23. van Vugt JL, Coebergh van den Braak RR, Lalmahomed ZS, Vrijland WW, Dekker JW, Zimmerman DD, et al. Impact of low skeletal muscle mass and density on short and long-term outcome after resection of stage I-III colorectal cancer. Eur J
Surg Oncol. 2018;44:1354-60.

24. Hall JC. Nutritional assessment of surgery patients. J Am Coll Surg. 2006;202:837-43.

25. Prado CM, Heymsfield SB. Lean tissue imaging: a new era for nutritional assessment and intervention. JPEN J Parenter Enteral Nutr. 2014;38:940-53.

26. Black D, Mackay C, Ramsay G, Hamoodi Z, Nanthakumaran S, Park KG, et al. Prognostic value of computed tomography: measured parameters of body composition in primary operable gastrointestinal cancers. Ann Surg Oncol. 2017;24: 2241-51.

27. Sueda T, Takahasi H, Nishimura J, Hata T, Matsuda C, Mizushima T, et al. Impact of low muscularity and myosteatosis on long-term outcome after curative colorectal cancer surgery: a propensity score-matched analysis. Dis Colon Rectum. 2018; 61:364-74.

28. Malietzis G, Currie AC, Athanasiou T, Johns N, Anyamene N, Glynne-Jones R, et al. Influence of body composition profile on outcomes following colorectal cancer surgery. Br J Surg. 2016;103:572-80.

29. Padilha CS, Marinello PC, Galvao DA, Newton RU, Borges $\mathrm{FH}$, Frajacomo F, et al. Evaluation of resistance training to improve muscular strength and body composition in cancer patients undergoing neoadjuvant and adjuvant therapy: a meta-analysis. J Cancer Surviv. 2017;11:339-49.

30. Ewaschuk JB, Almasud A, Mazurak VC. Role of n-3 fatty acids in muscle loss and myosteatosis. Appl Physiol Nutr Metab. 2014;39:654-62. 